\title{
Efficient Routing Protocol in the Mobile Ad-hoc Network (MANET) by using Genetic Algorithm (GA)
}

\author{
Hussein A. Lafta ${ }^{1}$ and Ahmed M. M. S. Al-Salih ${ }^{2}$ \\ 1 Computer Science Dept., Assistant Dean of the College of Science for Women, College of Science for Women, \\ University of Babylon, (Iraq). \\ 2 Information Netwoks Dept., College of Information Technology, University of Babylon, (Iraq).
}

\begin{abstract}
An Ad hoc network is a collection of wireless mobile hosts forming a temporary network without the aid of any centralized administration or standard support services. MANET can be defined using unstable network infrastructure, self-organizing network topology and independent node mobility. This becomes obtainable due to their routing techniques; in other terms, routing is a backbone for MANET. However, due to network load routing performance of MANET is degraded thus, some optimization on network routing strategy is required.

In this paper, we introduce a new technique by using the concept of Genetic algorithm (GA) with $A O D V$ Protocol to make routing decision in computer network.

The goal of this paper is to find the optimal path between the source and destination nodes and increased the QoS and Throughput. We implemented and compare this a new technique with the traditional AODV, and we shows that the new technique is better performance than the traditional AODV.
\end{abstract}

Keywords: AODV Routing, DSR Routing, Routing protocols, Genetic Algorithms, MANET, Multicast, Multihop, WSN protocols.

\section{Introduction}

Mobile ad-hoc networks (MANET'S) are decentralized, self-organizing networks capable of forming a network without relying on any fixed infrastructure, these networks allow spontaneous formation and deformation of mobile networks . Defining on ad hoc network as an autonomous system of mobile hosts connected by wireless links. A routing algorithm should strive to find a shortest path for transmission packet.ad hoc network features are mobility and flexibility, peer-to-peer multi-hop networks. Routing in mobile ad hoc networks depends on many factors for topology; find optimal path selection of routers.ad hoc network uses two different types of protocols. They protocols are Proactive and Reactive protocols.

Proactive protocol in a MANET should track of routes in all nodes destination. This protocol maintains routing table information and also called table-driven protocol. (Example of important proactive protocols for destination sequenced distance vector (DSDV), wireless routing protocol (WRP). In reactive protocols a node defining to route discovery process and also called on demand distance vector routing. The protocols are two types of mechanisms of "route discovery" and "route maintenance". (Example of reactive protocol for Dynamic Source Routing Protocol (DSR) and Ad-hoc On-Demand Distance Vector (AODV) [1].

Shortest path prblem in MANETS asks for the computation of path from source to destination node that minimizes the sum of total cost associated with the path. Several traditional algorithms like Bellman ford Algorithm, Dijkstraes Algorithm are developed to find the shortest path. These algorithms are not suitable for MANETS, but are suitable only for wired networks. For wireless networks, biologically inspired algorithms like Genetic Algorithm (GA), Particle Swarm Optimization (PSO) and Evolution Algorithm (EA) can be implemented efficiently. Genetic Algorithm is an adaptive search algorithm based on natural evolution and genetics. Major steps involved here are generation of population of solutions, using fitness function and application of genetic operators.

Genetic algorithm is applied with different routing protocols. The different routing protocols are Ad hoc On demand Distance Vector (AODV), Ad hoc On demand Multipath Distance Vector(AOMDV), Dynamic Source Routing(DSR), Destination Sequenced Distance Vector Routing (DSDV). Genetic Algorithm when applied with AODV provides the best results in terms of performance.

This paper proposed for solving the optimal (Best) path routing problem using genetic algorithm. Genetic algorithm is a programming methods and evaluation of problem solving method. The genetic algorithm then evaluates each candidate to fitness function. This algorithm is best of the searching algorithm. The proposed genetic algorithm using best optimal path between source and destination nodes in ad hoc networks and evaluate of fitness function for cost and bandwidth. 


\section{Background And Related Work}

Ad hoc routing protocols can be divided into two categories: topology based and position based. Topology based routing protocols use the information about the links that exists in the network to perform packet forwarding. Position-based routing protocols use the geographical position of nodes to make routing decisions, which results in improving efficiency and performance. In recent developments, position-based routing protocols exhibit better scalability, performance and robustness against frequent topological changes [2]

Topology-based routing can be further divided into two approaches: Proactive and reactive approach. Proactive routing protocols periodically broadcast control messages in an attempt to have each node always know a current route to all destinations. Proactive approach maintains routing information about the available paths in the network even if these paths are not currently used. But the drawback of this approach is that the maintenance of unused paths. Reactive routing protocols maintain only the routes that are currently in use thereby reducing the burden on the network, are more appropriate for wireless environments because they initiate a route discovery process only when data packets need to be routed. There is no periodic routing packet required. The destination sequenced distance vector and the wireless routing protocol are popular examples of table driven protocols. Dynamic source routing, on demand distance vector routing and associatively-based routing is representative on demand (reactive) protocol.

Almost every wireless network nodes communicate to base-station and access points there by cooperating to forward packets hop by-hop. Wireless ad hoc networks can be further classified as :

a. Mobile Ad hoc Networks (MANETs)

b. Wireless Mesh Networks (WMNs)

c. Wireless Sensor Networks (WSNs)

Mobile Ad hoc networks (MANETS) consists of mobile platforms, which are free to move arbitrarily. These are self-organizing and adaptive networks. These networks allow spontaneous formation and deformation of mobile networks. A MANET is an autonomous collection of mobile users that communicate over relatively bandwidth constrained wireless links. Since the nodes are mobile, the network topology may change rapidly and unpredictably over time. The network is decentralized, where all network activity including discovering the topology and delivering messages must be executed by the nodes themselves i.e., routing functionality will be incorporated into mobile nodes.

In [3]. C.Mala, et al proposed a PSO Based Multicast Routing Algorithm with Bandwidth and Delay as the Quality of Service Constraints for optimization. In [3] Liu Jing et al have addressed the Quality of Service Multicast routing problem using Particle Swarm Optimization with the Quality of Service Parameters under consideration were Cost ,Delay, Delay Jitter and Packet Loss. In [3].C.Mala, et al discuss solving the Multicast Routing problem with Quality of Service parameters Buffer Space and Queuing Delay in addition to the other basic Quality of Service Parameters using Particle Swarm Optimization. The next section discusses the proposed work and the algorithm for solving the problem under study. The simulation and results are discussed in the Simulation and performance analysis section, followed by conclusion. Each node selectively adds only the best (most fit) nodes in its neighbourhood to its proactive region. In [4] Jihar Doshi et al, the adjustment of the zone is based on an approximation cost model. [4] Adjusts the proactive region in order to make a node more accessible. We change the Proactive region in order to reduce route acquisition latencies. Unlike [4], we use the concept of FITNESS (a Genetic Algorithm-based technique) to determine the node's participation in proactive routing. This yields a more realistic proactive region as it takes into account the changing environment of a node.

\section{Routing Protocols For Mobile Ad-Hoc Network (Manet)}

MANET routing protocols are typically subdivided into two main categories: proactive routing protocols and reactive on-demand routing protocols [5]. Proactive routing protocols are derived from legacy Internet distance-vector and link-state protocols. They attempt to maintain consistent and updated routing information for every pair of network nodes by propagating, proactively, route updates at fixed time intervals. As the routing information is usually maintained in tables, these protocols are sometimes referred to as TableDriven protocols.

Reactive on demand routing protocols, on the other hand, establish the route to a destination only when there is a demand for it. The source node through the route discovery process usually initiates the route requested. Once a route has been established, it is maintained until either the destination becomes inaccessible (along every path from the source), or until the route is no longer used, or expired [5,6].

REACTIVE ROUTING PROTOCOLS:- These protocols depart from the legacy Internet approach. To reduce the overhead, the route between two nodes is discovered only when it is needed. Representative reactive routing protocols include:

Dynamic Source Routing (DSR), Ad hoc On Demand Distance Vector (AODV), Temporally Ordered Routing Algorithm (TORA), Associativity Based Routing (ABR), Signal Stability Routing (SSR). DSR is a 
loop-free, source based, on demand routing protocol [7], where each node maintains a route cache that contains the source routes learned by the node. The route discovery process is only initiated when a source node do not already have a valid route to the destination in its route cache; entries in the route cache are continually updated as new routes are learned. Source routing is used for packets_forwarding. AODV is a reactive improvement of the DSDV protocol.

AODV minimizes the number of route broadcasts by creating routes on-demand [3], in the DSDV algorithm. Similar to DSR, route discovery is initiated on-demand, the route request is then forward by the source to the neighbors, and so on, until either the destination or an intermediate node with a fresh route to the destination, are located. DSR has a potentially larger control overhead and memory requirements than AODV since each DSR packet must carry full routing path information, whereas in AODV packets only contain the destination address. On the other hand, DSR can utilize both asymmetric and symmetric links during routing, while AODV only works with symmetric links (this is a constraint that may be difficult to satisfy in mobile wireless environments).

In addition, nodes in DSR maintain in their cache multiple routes to a destination, a feature helpful during link failure. In general, both AODV and DSR work well in small to medium size networks with moderate mobility.

TORA is another source-initiated on-demand routing protocol built on the concept of link reversal of the Directed Acyclic Graph (ACG) [8]. In addition to being loop-free and bandwidth efficient, TORA has the property of being highly adaptive and quick in route repair during link failure, while providing multiple routes for any desired source/destination pair. These properties make it especially suitable for large, highly dynamic, mobile ad hoc environments with dense nodes_ populations. The limitation in TORA_s applicability comes from its reliance on synchronized clocks. If a node does not have a GPS positioning system, or some other external time source, or if the time source fails, the algorithm fails.

ABR protocol is also a loop free protocol, but it uses a new routing metric termed degree of association stability in selecting routes, so that route discovered can be longer-lived route, thus more stable and requiring less updates subsequently. The limitation of ABR comes mainly from a periodic beaconing used to establish the association stability metrics, which may result in additional energy consumption. Signal Stability Algorithm (SSA) [9] is basically an ABR protocol with the additional property of routes_selection using the signal strength of the link.

In general, on-demand reactive protocols are more efficient than proactive ones. On-demand protocols minimize control overhead and power consumption since routes are only established when required. By contrast, proactive protocols require periodic route updates to keep information current and consistent; in addition, maintain multiple routes that might never be needed, adding unnecessary routing overheads.

Proactive routing protocols provide better quality of service than on-demand protocols. As routing information is constantly updated in the proactive protocols, routes to every destination are always available and up-to-date, and hence endto-end delay can be minimized. For on-demand protocols, the source node has to wait for the route to be discovered before communication can happen. This latency in route discovery might be intolerable for real-time communications.

\section{Genetic Algorithms:}

Genetic algorithms are adaptive heuristic search algorithm premised on the Darwin's evolutionary ideas of natural selection and genetic. The basic concept of genetic algorithms is designed to simulate processes in natural system necessary for evolution [Pratibha Bajpai 2010]. Strength of Genetic Algorithm comes from :[10]:

1. Genetic algorithms are parallel. They can explore the solution space in multiple directions at once. So, convergence to an optimal solution does not depend on the chosen initial solution.

2. They are well-suited to solving problems having huge search space.

3. They perform well in problems for which the fitness landscape is complex - where the function is discontinuous, noisy, changes over time, or has many local optima.

\section{IV.1 How Genetic Algorithms Work:}

Genetic algorithm maintains a population of individuals, for generation. Each individual represents a potential solution to the problem. Each individual evaluated to give some measure of its fitness. Some individuals undergo random transformations by means of genetic operations to form new individuals. There are two type of transformation [11]:-

1. Mutation, which forms new individuals by making changes in a single individual.

2. Crossover, which forms new individuals by combining parts from two individuals.

The new individuals, called offspring, are then evaluated. A new population is formed by selecting the more fit individuals from the parent population and offspring population transformation [Muhammad T. et al, 2009]. 
After several generations, genetic algorithm converges to the best individual, which represents an optimal or suboptimal solution to the problem. The block diagram of a GA cycle is presented in Figure 1 Steps of The Genetic Algorithm as follows:

1. [Start] Generate random population of $\mathrm{n}$ chromosomes (suitable solutions for the problem).

2. [Fitness] Evaluate the fitness $f(x)$ of each chromosome $x$ in the population.

3. [New population] Create a new population by repeating following steps until the new population is complete.

i. [Selection] Select two parent chromosomes from a population according to their fitness (the better fitness, the bigger chance to be selected).

ii. [Crossover] With a crossover probability cross over the parents to form a New offspring (children). If no crossover was performed, offspring is an exact copy of parents.

iii. [Mutation] With a mutation probability mutate new offspring at each Locus (position in chromosome).

iv.[Accepting] Place new offspring in a new population.

4. [Replace] Use new generated population for a further run of algorithm.

5. [Test] If the end condition is satisfied, stop, and return the best solution in current population.

6. [Loop] Go to step 2.

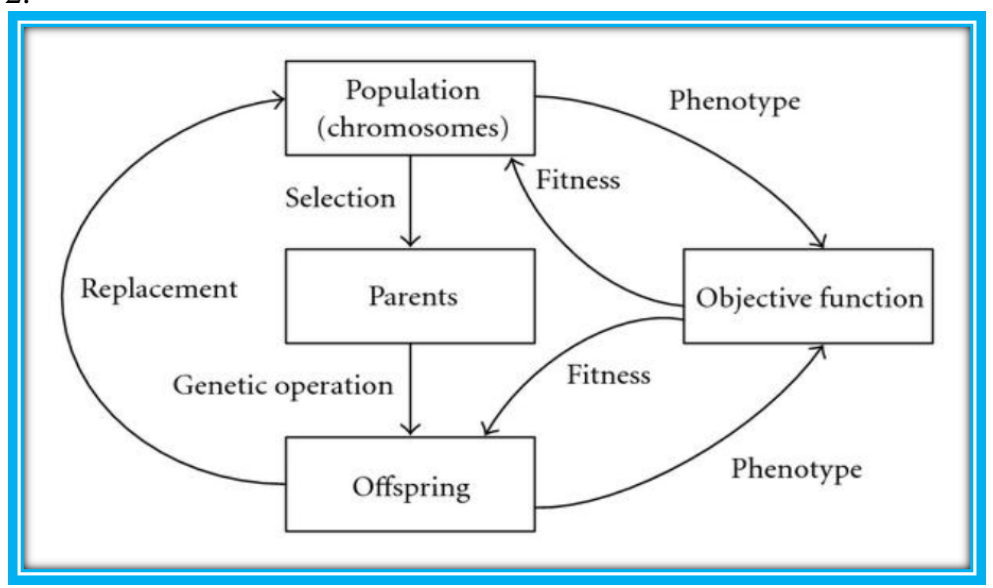

Fig. 1: The Block Diagram of Genetic Algorithms

\section{IV.2 Encoding:}

A suitable encoding is found for the solution to problem so that each possible solution has unique encoding and the encoding is some form of a string.

Many encoding techniques have been created for particular problems to provide effective representation of genetic algorithms. According to what kind of a gene, the encoding methods are the following:

1. Binary encoding.

2. Real-number encoding.

3. Integer or literal permutation encoding.

\section{IV.3 Genetic Algorithms Cycle:}

Each cycle in GA produces a new generation of possible solutions for a given problem.

\section{1) Initialization:}

Initially, many individual solutions are randomly generated to form an initial population. The population size depends on the nature of the problem, but typically contains several hundreds or thousands of possible solutions. Traditionally, the population is generated randomly, covering the whole range of possible solutions (the search space). Occasionally, the solutions may be "seeded" in areas where optimal solutions are likely to be found [9].

\section{2) Fitness:}

The performance of the strings, often called fitness, is then evaluated with the help of some functions, representing the constraints of the problem.

\section{3) Selection:}

Selection operation is the main operation in genetic algorithm. It is used to choose the best fit individuals in the population to create the new individuals. New individuals will participate in further genetic operations to create the next generation of population. The next generation of population is created with a hope to reach the optimal solution [12].

Many selection methods have been proposed. The most common types are :

1. Roulette wheel selection. 
2. Rank selection.

3. Tournament selection.

4. Elitism

\section{IV.4 AD HOC NETWOK WITH GENETIC ALGORITHM}

Ad hoc network can represented as a connected graph with $\mathrm{N}$ nodes. The optimization metric is cost of path between the source and destination. The sum of cost of individual hops is total cost.

The goal is to find the path with minimum total cost between source and destination node using genetic algorithm with simple and effective manner.

The path from source to destination node is a feasible solution. Beginning a random population of strings is generated, which represents feasible or unfeasible solutions. Unfeasible solutions are strings that can't reach the destination. A Chromosome corresponds to possible solution of the optimization problem. Thus each chromosome represents a path which consists of sequences of positive integers that represent IDs of nodes through which a routing path passes with source node followed by intermediate node to destination node. The default maximum chromosome length is equal to the number of nodes.

\section{Simulation Model}

The network simulator ns2 was used as a simulation means; it is a program product with an open code that combines the network simulator, various protocol implementation and visualization means, traffic model means, etc.

Ns2 is an object-oriented software and its core is implemented in $\mathrm{C}++$. OTcl (Object oriented Tool Command Language) is used as a simulation scenario language. Ns2 supports entirely the C++ classes' hierarchy and detailed OTcl classes' hierarchy. Both hierarchies have the same structure, i.e., there is a singlevalued correspondence between the same classes of the two hierarchies. The usage of two programming languages in ns 2 is explained by the requirement to use a system language for the detailed protocol simulation and also by the ease of operating the higher abstraction level programming language to realize and modify various simulation models. Such an approach is a compromise between the ease of operation and velocity.

The using of ns 2 makes it possible to describe the network topology, traffic sources and receivers configuration, connection parameters (the passband, the delay, the packet loss probability and many other simulated system parameters. In the course of simulation it is possible to control buffer parameters, to monitor received, transmitted and lost packets, to gather statistics and etc. The output trace-file generation provides information on the traffic dynamics, connections state and network object state as well as protocol operation.

The simulator uses an interpreter which is otclsh command shell add-on with the using of command and configuration interface in OTcL language (that is an object-oriented version for TcL, Tool Command Language) In this case simulation is implemented by means of OTcL scenarios/scripts.

Packet delivery fraction: The ratio of the data packets deliv-ered to the destinations to those generated by the CBR sources.

Average end to end delay: Average end to end delay is an average end to end delay of data packets that is average time needed to transfer a data packet from source to destination. Once the difference between every CBR packets sent and re-ceived is found, it is divided by the total number of CBR pack-ets received. This gives the average end to end delay for re-ceived packets. The lower is the end to end delay, the better the application performs.

$$
\text { E2E Delay }=\sum(C B R r c v \text { Time }-C B R \text { sentTime }) / \sum C B R r c 0
$$

Normalized routing load: It is defined as the total number of routing packets transmitted per data packet. It is calculated by dividing the total number of routing packets sent (includes forwarded routing packets as well) by the total number of data packets received.

$$
\mathrm{NRL}=\sum R T R p k t s / \sum C B R r C V
$$

\section{Implementation And Results}

To simulate the research work we working around various software simulation network tools, and we found the NS2 network simulator which is a discrete event network simulator where all the participating devices can be visualize by the nodes and the corresponding network utilities can be installable. Moreover it simulation required additional script writing for performing the experiments using $\mathrm{C}++$ scripts. User has freedom to select their own language for scripting. Once network script is compiled and executed some additional files are generated to get the network information for performance evaluation and animation of network and created scenario. For visualization NETANIMATOR (NAM) can be used and for performance graph and results is a good utility for use. 


\section{VI.1 Simulation setup}

To simulate our proposed work we first setup network environment. Then we simulate and compare our proposed techniques in the two scenarios.

\begin{tabular}{|c|c|}
\hline Parameters & Description \\
\hline Number of node & $10,20,30,40,50,60,70,80,90,100$ \\
\hline Channel type & Wireless Channel \\
\hline Network interface type & WirelessPhy \\
\hline MAC type & Mac/802_11 \\
\hline Time of simulation & AODEC S \\
\hline Routing protocol & 1000 x 1000 \\
\hline Simulation size & OmniAntenna \\
\hline Antenna Model & AODV \\
\hline Routing Protocol & \\
\hline
\end{tabular}

Network simulation having two most important parts first parameters required for network simulation and second the simulation of network scenario for performing the experiments and results evaluation.

1. Implementation of MANET using AODV protocol: in this network scenario we implement a network using AODV routing protocol over variable number of nodes in network.

2. Implement AODVwith GA and measure their performance: here we implement and modify the network protocol and implement GA in traditional AODV protocol.

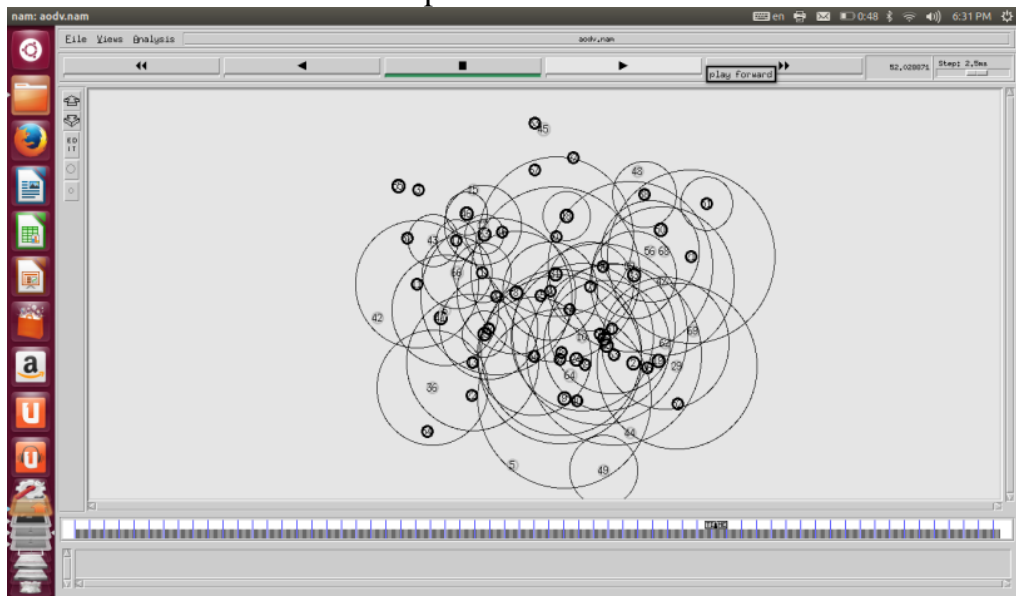

Fig. 2 shows the animation and propagation

Throughput provides the efficiency of the system and given using the above given fig. after evaluation of the performance parameters we can see that the overall performance of the proposed GA is much efficient than the traditional routing protocol AODV Figure 3 .

This section of presented paper provides the performance analysis of the proposed routing protocol with respect to the AODV routing protocol. For performance analysis, we use more than one performance parameters.

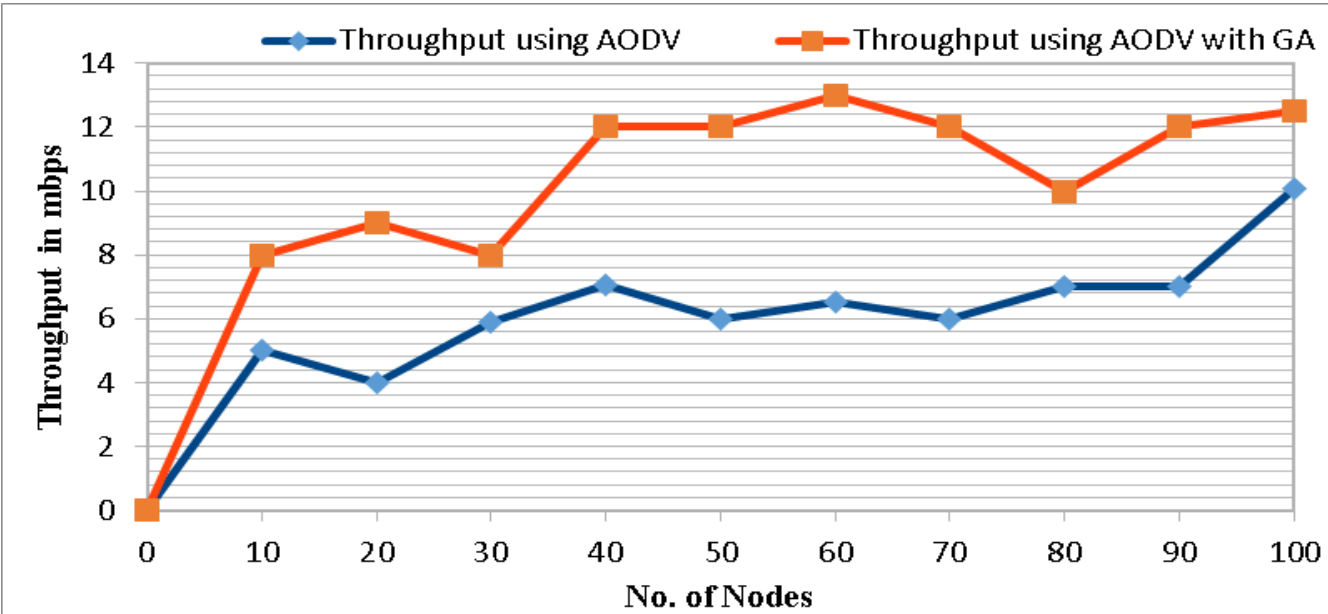

Fig. 3 Shows the Throughput

Packet delivery ratio provides information about the performance of any routing protocols. Where PDR is 
estimated using the formula given

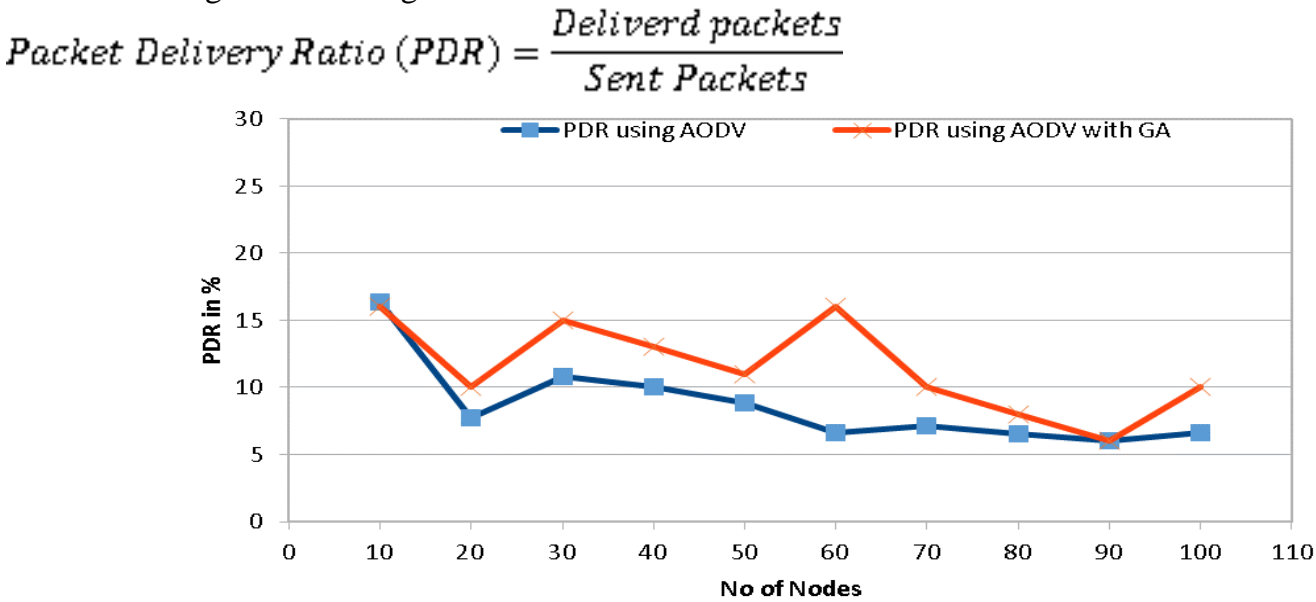

Fig. 4 Shows the Packet Delivery Ratio

Total time difference between send and receiving of any data packets is known as end to end delay. Fig shows the end to end delay between both protocols.

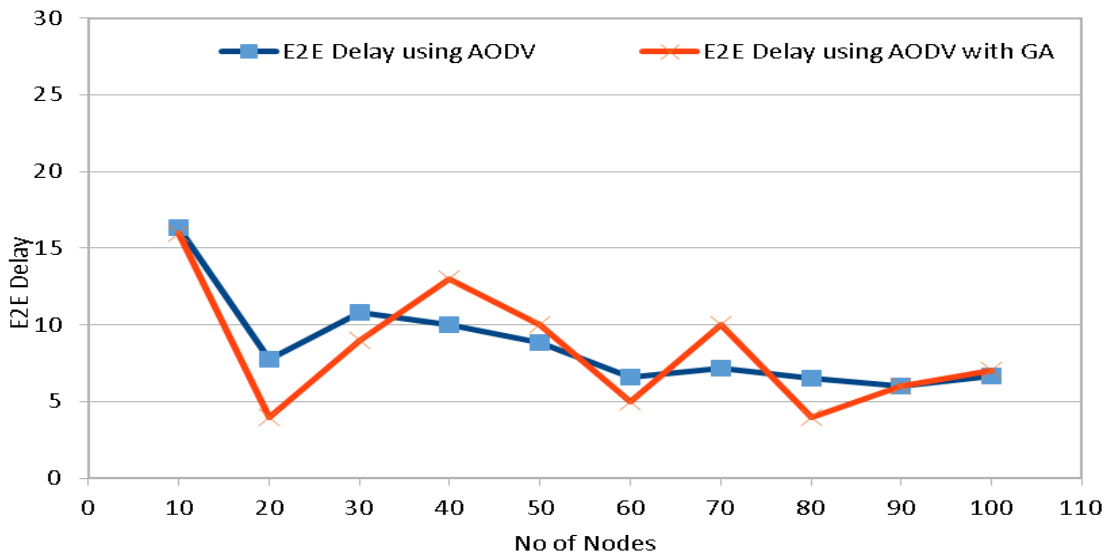

Fig. 5 Shows End to End Delay

\section{Conclusion}

This work presents a routing protocol for mobile ad hoc network using genetic algorithm. The proposed scheme for genetic algorithm to find the optimal path from source and destination nodes. we provide the complete research work and there implementation using NS2 network simulator and visualize using the NAM tool. Here given simulation is based on two different routing technique in MANET using simple or traditional AODV routing and an enhanced routing using Genetic Algorithm (GA) technique, and results are evaluated after performance analysis we found that GA better performance than traditional routing.

The GA optimal routes suggest that the complexity of having smart routers that are able to forward packets onto each branch at a different rate. However, it is hard to draw due dynamic topology of source-destination pair selections. In Mobile ad hoc networks, the unstable transport layer and inhibited amount of traffic being carried out by the network, which owes high packet loss rates and frequent topological changes.

\section{References}

[1]. E.M. Belding-Royer, C.-K. Toh, A review of current routing protocols for ad-hoc mobile wireless networks, IEEE Personal Communications Magazine (April 1999) 46-55.

[2]. Anju Sharma, Madhavi Sinha, "A Differential Evaluation Algorithm For Routing Optimization In Mobile Ad Hoc Networks", The Birla Institute Of Technology, Jaipur Mesra, Ranchi Campus Jaipur, Rajasthan, International Journal Of Computer Science And Network (IJCSN),Volume 1, Issue 4, Www.Ijcsn.Org ISSN 2277-5420,Pages 109-115, August 2012.

[3]. C.Mala, A.Anurag Mahesh ,R.Aravind ,R.Rajgopal ,Narendran Rajagopalan , B.Nithya , "Simulated Study Of Qos Multicast Routing Using Genetic Algorithm", National Institute Of Technology,Tiruchirappalli. World Applied Programming, Vol (2), Issue (5),pages 342-348,Issn: 2222-2510@2011 Wap Journal. Www.Waprogramming.Com, May 2012.

[4]. Jihar Doshi, Prahlad Kilambi, "Safar: An Adaptive Bandwidth-Efficient Routing Protocol For Mobile Ad Hoc Networks", Sri Venkateswara College Of Engineering, University Of Madras, Pennalur, Sriperumbudur, 602105Jigar@Doshi.Com, Prahlad@ Acm.Org. S. Pierre, M. Barbeau, and E. Kranakis (Eds.): Adhoc-Now 2003, Lncs 2865, Pp. 12-24, 2003. C SpringerVerlag Berlin Heidelberg 2003. 
[5]. Perkins C.E., E.M. Royer, Ad-hoc on-demand distance vector routing, in: Proceedings of 2nd IEEE Workshop on Mobile Computing Systems and Applications, February 1999.

[6]. Elizabeth Belding-Royer, Routing approaches in mobile ad hoc networks, in: S. Basagni, M. Conti, S. Giordano, I. Stojmenovic (Eds.), Ad Hoc Networking, IEEE Press Wiley, New York, 2003.

[7]. Y. Sankarasubramaniam, O. Akan and L. Akyildiz, IESRT: Event-to-Sink Reliable Transport in Wireless Sensor Networks," in MobiHoc'03, Annapolis, Maryland, USA, June 2003, pp. 113-112.

[8]. V.D. Park, M.S. Corson, A highly adaptive distributed routing algorithm for mobile wireless networks, in: Proceedings of INFOCOM 97, April 1997.

[9]. Pratibha B. 2010 Pratibha B. and Manoj K., " Genetic Algorithm - an Approach to Solve Global Optimization Problems", Indian Journal of Computer Science and Engineering, Vol. 1 No. 3 pp. 199-206, 2010.

[10]. Amol C. A. 2011 Amol C. A. and Rajankumar B., " Hybrid Genetic Algorithmic Approaches for Personnel Timetabling and Scheduling Problems in Healthcare", International Conference on Technology Systems and Management (ICTSM), 2011.

[11]. Muhammad T. 2009 Muhammad T. and M. A. Abido, " Assessment of Genetic Algorithm Selection, Crossover and Mutation Techniques in Reactive Power Optimization", IEEE, 2009.

[12]. Rakesh K. 2012 Rakesh K. and Jyotishree, "Effect Of Polygamy With Selection In Genetic Algorithms", International Journal of Soft Computing and Engineering (IJSCE) ISSN: 2231-2307, Vol. 2, 2012. 